\title{
A computer laboratory program in engineering mathematics to enhance mathematical conceptualisation
}

\author{
Raymond Summit ${ }^{1}$
}

(Received 31 January 2010; revised 13 May 2010)

\begin{abstract}
This article describes a laboratory supplementary program that was integrated into a traditional mining engineering mathematics unit. The practical classes consisted of computer investigations designed to help develop mathematical concepts. The program described here was mainly web based and did not directly rely on a computer algebra system for its implementation. An evaluation of the program is included.
\end{abstract}

\section{Contents}

1 Introduction

C281

2 Approaches to learning mathematics

C282

3 Establishing a laboratory program

C283

http://anziamj . austms.org.au/ojs/index.php/ANZIAMJ/article/view/2616 gives this article, (c) Austral. Mathematical Soc. 2010. Published June 2, 2010. ISSN 1446-8735. (Print two pages per sheet of paper.) 
4 The laboratory program at WASM

C285

5 Evaluation and discussion

C292

6 Conclusion

C296

References

C297

\section{Introduction}

This article discusses a program of laboratory classes based on investigations that used a computer as a tool to explore mathematical concepts. Since the program used multi/hypermedia resources, it was not dependent on a particular computer algebra system (CAS). The laboratory program was implemented as part of a first year mining engineering mathematics unit at the Western Australian School of Mines (WASM).

Prior to the introduction of the laboratory program, students at WASM followed a traditional program in mathematics, with lectures and tutorials only. It had become apparent that our students were focusing on learning the procedures needed to answer examination questions in order to pass. They showed little or no aspiration to actually understand the mathematical concepts so that their acquired knowledge could be used in unfamiliar situations.

Hallet [5] acknowledged that simply memorising algorithms is largely a wasted effort, since the algorithms are soon forgotten. She further asserted that, left unchecked, courses that support such behaviour perpetuate the idea that mathematics involves doing calculations and following recipes, rather than thinking. To encourage deeper learning, a laboratory program that allowed students to explore and cultivate understanding of mathematical concepts was developed. The implementation of this program is discussed. 


\section{Approaches to learning mathematics}

Relevant to the learning of mathematics is the notion of deep and surface learning. A deep or holistic approach to learning occurs when a student tries to make sense of new information and relate it to what he or she already knows. A surface or atomistic approach reduces learning to rote memorisation [6].

The call for greater conceptual understanding in calculus can be traced to the calculus reform movement which developed in the mid 1980s. The movement sought "to find creative ways to help students gain a deeper understanding of the ideas of calculus" [1, p.1]. The traditional approach to teaching and learning calculus left gaps in conceptual understanding [3]. The aim of the reform movement was to trim content and to deepen students' level of understanding of the core concepts [8], to make calculus more meaningful and to shift it from being "rote memorisation and symbol manipulation" [3, p.165]. The emphasis in reform courses moved away from manipulative skills, towards mathematical explorations and applications, often overlooked by classical approaches [16].

Studies have reported greater understanding when students received explanations in both visual and verbal formats $[7,11]$. With the development of computer systems it has become increasingly possible to incorporate and swap between the visually rich geometric and numerical approaches and the algebraic [10]. Cunningham asserted that through visualisation, "students not only learn mathematics but also learn new ways to think about and do their own mathematics" [1, p.70], thus promoting a deeper learning.

However, Habre [4] reported that students were generally more comfortable with the algebraic approach because they were familiar with it from their prior traditional schooling. There is a need to expose students to the geometric approach before they can become more adept at thinking visually. When students are provided with the opportunity to practise, they come to appreciate the power of the visual approach and its usefulness as a tool in 
solving difficult problems [4].

A number of studies recommended a balance between visual, numerical and analytical approaches $[5,14]$. The algebraic approach has firmly established its place in the curriculum. To enhance understanding of concepts, visual and numerical approaches also need to be included.

Thus we decided that a review of the first year engineering mathematics curriculum was necessary to avail our students of the many benefits possible with the use of computer technology. The traditional algebraic approach was to be retained in the curriculum within the lecture and tutorial programs. However, wherever appropriate, visual cues were to be included in these sessions, in order to enhance understanding. To further develop greater conceptual understanding of mathematics, a laboratory program was to be developed to emphasise the visual, geometric and numerical approaches. This program aimed to supplement the existing lecture program by providing students with an opportunity to attain a deeper understanding of mathematical concepts with the use of computer technology.

\section{Establishing a laboratory program}

We first introduced a computer laboratory program at the beginning of 2006 . Since maths classes were timetabled for five hours per week, it was possible to allow three hours for lectures, one hour for tutorials and one hour for laboratory classes.

The initial laboratory program comprised worksheets to be completed in Maple, a CAS. The aim of the program was to expose students to some of the capabilities of the software and to build skills in its use. Suitable resources were sourced and used within the program. A good example, entitled "Vector Algebra, Lines and Planes", can be found at Brooks/Cole's 
companion website. ${ }^{1}$ The worksheet provides scripts for performing vector algebra and drawing lines and planes, so that students can see how these tasks are done using Maple. It starts with a review of the key concepts that are used in the worksheet and is well formatted with clear documentation. Its objective is to illustrate how Maple can be used to perform vector algebra and to generate the equations of lines and planes.

We believed that it was worthwhile to expose mathematics students to Maple and to skill them in its use. However, our students indicated displeasure with having to learn CAS skills that they were unlikely to use in their mining engineering vocation. In addition to this, students felt that the laboratory program did not help them prepare for examinations. This view was backed up by Tonkes et al. [13] and Pemberton [9]. Syntax can be a major source of frustration when using a CAS [9] and often the mathematics is lost in trying to negotiate the software [13]. Vlachos and Kehagias [16] pointed out that the time required for students to develop the computer skills necessary to exploit the capabilities of a CAS, created an overhead that could not be ignored.

It was time for our laboratory program to take a directional change away from learning Maple programming, towards mathematical investigations. This change in focus aimed to provide activities that developed understanding of concepts. Cases have been reported of laboratory programs where students were eased of the burden of learning a CAS by being given the required computer code $[2$, e.g.]. It was possible to implement this desired directional change in our laboratory program by providing the code required for mathematical investigations within a CAS environment. This required setting up a completely new program.

With ever increasing demands placed on academics and dwindling availability of resources, it is becoming increasingly difficult to embark upon the review and improvement of curriculum. Cunningham pointed out that "the graphics

\footnotetext{
${ }^{1}$ http://www.brookscole.com/math_d/special_features/maplelabs05/maple_ 9-5_revisions_penna/11-geometryinspace/p02a.pdf
} 
... and sophisticated user interaction needed for effective educational software require a great deal of work to program" [1, p.68] and that professional programmers need a year or longer to become proficient. Hence, it seemed unviable to embark upon instituting a computer based laboratory program without having the necessary resources. The compact disk that accompanied the unit's textbook [12] provided a suitable multimedia resource for the desired laboratory program. At the transitional stage, some Maple worksheets were retained and some mathematical investigations using multimedia were introduced. Students responded favourably to the investigative explorations.

By 2008, our laboratory program was based solely on mathematical investigations. The focus of the program was on using visualisation to help develop and reinforce mathematical concepts. The new edition of the textbook offered the investigations freely online. ${ }^{2}$ The website is organised into modules that create visual stimuli for students to explore mathematical concepts. Each module includes a note about the concepts being explored, instructions and a set of questions to investigate. A more detailed look at the laboratory program is given in the next section.

\section{The laboratory program at WASM}

The program consisted of six fortnightly investigations over the twelve week semester. Laboratory classes met weekly for one hour with a tutor, where discussion was encouraged. Students were required to submit individual reports at the end of the fortnight. As some students can be more concerned about getting a good grade than acquiring subject knowledge (known as 'pseudo-learners' [15]), a substantial weighting of $20 \%$ of the final assessment was allocated to laboratory reports. Plagiarism was not evident, probably because laboratory classes were supervised. Students were encouraged to use the mathematical word processor capacity of Maple to write their reports.

${ }^{2}$ http://www.stewartcalculus . com/tec/ 
The main aim of the laboratory program was to provide students with an opportunity to further visually explore concepts introduced in lectures. To illustrate this visual approach, let us consider a typical problem found in calculus courses: "Find the traces of the surface with equation $x^{2}+y^{2}-z^{2}=1$ in the planes $x=n, y=n, z=n$. Identify the surface and sketch it."

The traditional approach would require students to work algebraically with the equation of the surface in order to find the equations of the traces. Many students struggle with the mathematics of the substitutions and may not recognise the equations of the traces as hyperbolas and ellipses. In contrast, the laboratory investigation provides a visual aid for the traces. Figure 1 shows that students can use a slider to move the $x=n$ plane through the surface to obtain traces. Students are asked to find the equations of the family of traces, thus tying the algebraic solution with a visual image. Critical values of $n$ can also be found visually. Students then investigate the traces of the surface in the planes $y=n$ and $z=n$. Further investigations of other surfaces are also provided.

In the second part of the investigation, students are given horizontal and vertical traces of surfaces, from which they can visualise the surface, and are asked to derive their equations.

The second investigation of our program allows students to explore the effect of changing the constants in the equation of the six basic types of quadric surfaces. Students are able to see how the shape of the surface changes when the constants in its equation are changed by adjusting their values using the sliders (Figure 2).

In the next investigation (Figure 3), Riemann sums are shown of the estimates of the area under a curve using the left, right and mid points of the intervals and using Simpson's and the trapezoidal rules. The estimated areas are compared to the exact areas to investigate when over, under or exact estimates are obtained. The effect of increasing the number of intervals is clearly demonstrated. The investigation explores the area under the curves of various functions. Conclusions can be reached about the relative accuracy 
- Traces of surface $x^{2}+y^{2}-z^{2}=1$
$\square \mathrm{x}=\mathbf{n}$
$\square \mathbf{y}=\mathbf{n}$
$\square \mathbf{z}=\mathbf{n}$
$\square x^{2}+y^{2}-z^{2}=1$
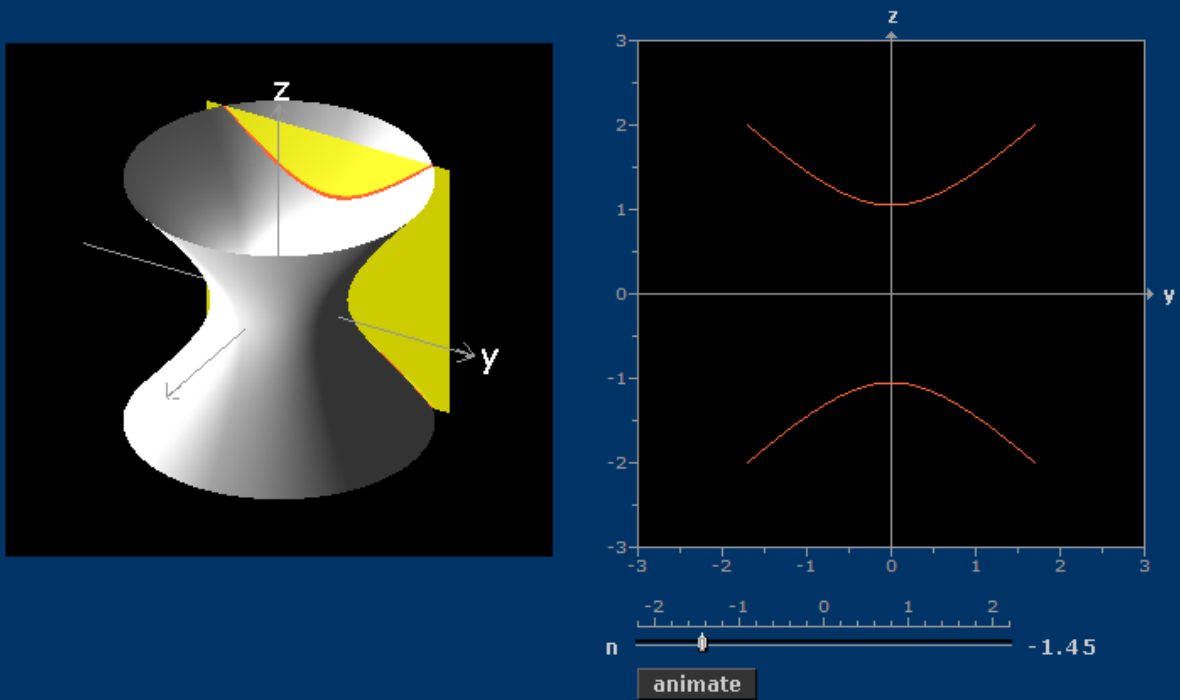

Figure 1: Traces of a surface 


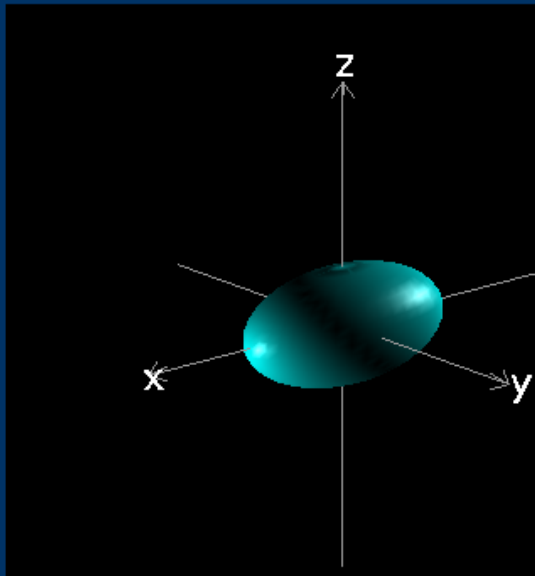

Click and drag to rotate axes
- Ellipsoid

$$
\frac{x^{2}}{a^{2}}+\frac{y^{2}}{b^{2}}+\frac{z^{2}}{c^{2}}=1
$$

a

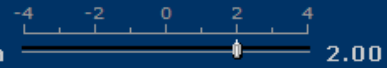

b
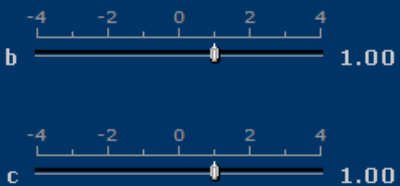

Figure 2: Quadric surfaces 


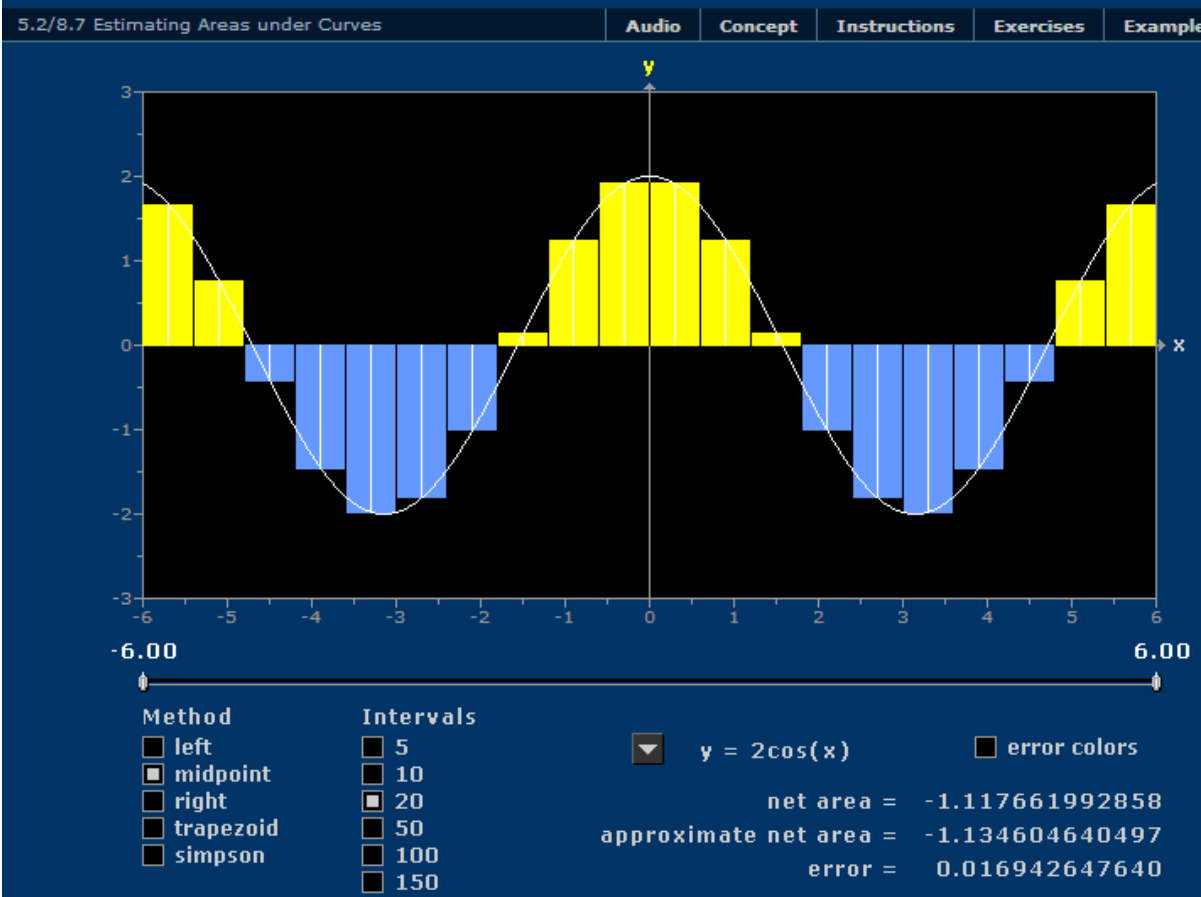

FiguRE 3: Estimating areas under curves

of the various methods.

Figure 4 shows an investigation which considers direction fields for a variety of first order differential equations of the form $d y / d x=F(x, y)$. Solution curves passing through various initial points are compared and a number of differential equations are presented. The existence of equilibrium solutions is investigated, as is the relationship between the curves in such cases. The construction of a direction field is a tedious process and requires numerous calculations. In practice, software is used to construct direction fields. This program frees the student to explore the characteristics of solution curves 


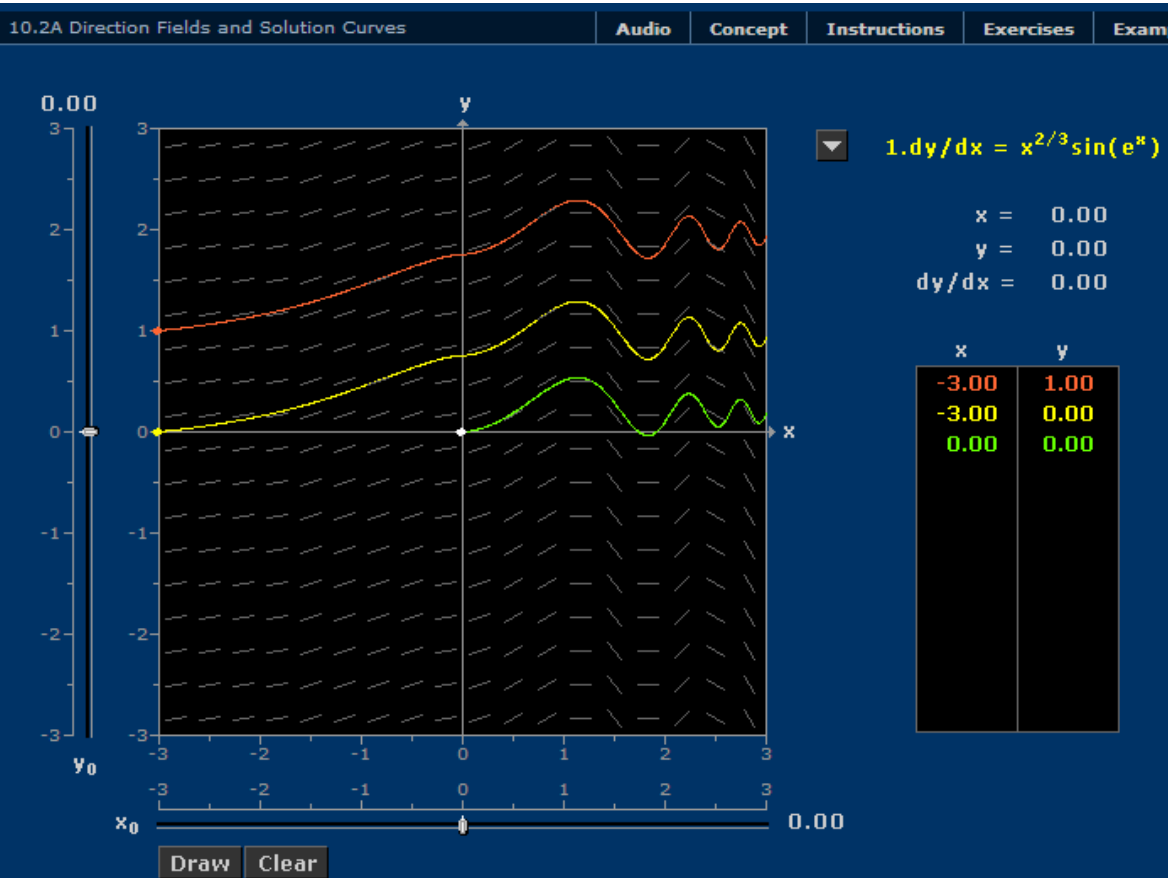

FiguRE 4: Direction fields and solution curves

without the tedium of constructing the direction fields.

In the fifth investigation, students are supplied with a sample spreadsheet (written by myself) illustrating the use of the Runge-Kutta and Euler methods to calculate estimates of a function given an initial value differential equation. The technique is taught in many calculus courses and is very labour intensive. Thus, it is not practical to perform the calculations without the aid of technology. In this investigation, students are asked to compare estimates obtained using both the Runge-Kutta and Euler methods for various step sizes. From the results, students are able to draw conclusions about the 

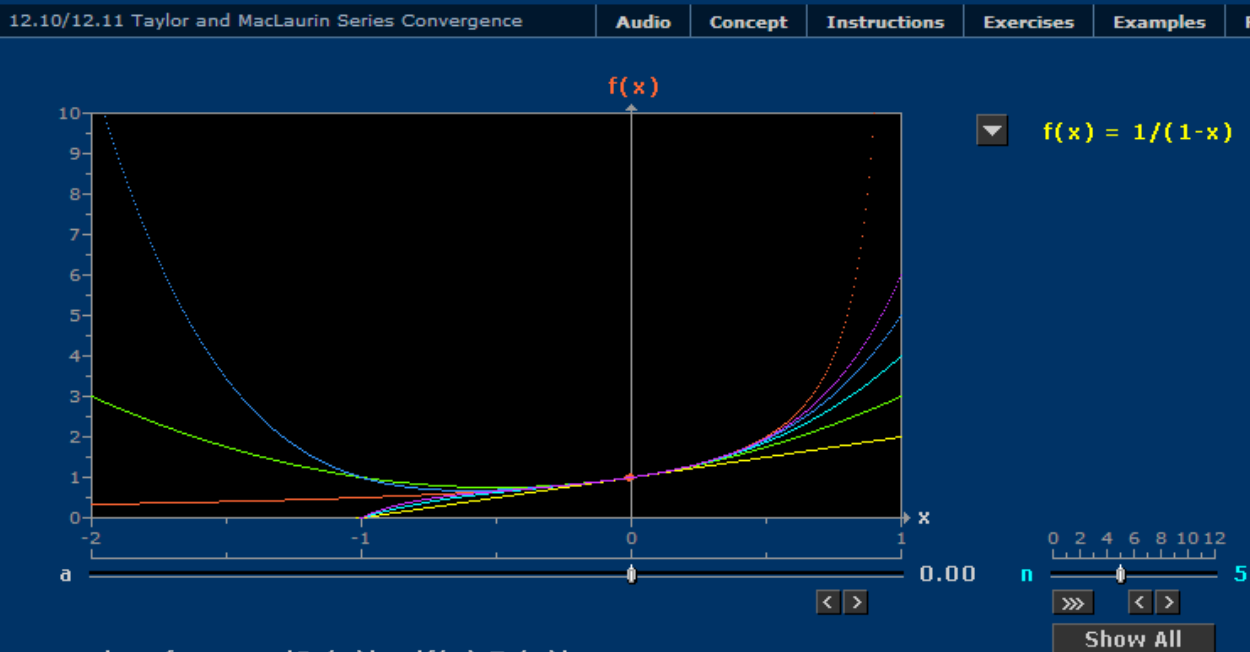

size of error $=\left|R_{n}(x)\right|=\left|f(x)-T_{n}(x)\right|$

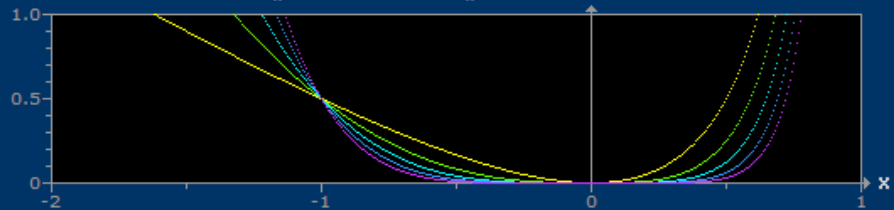

Show Series

FiguRE 5: Taylor and MacLaurin series convergence

accuracy of the two methods. Students are subsequently asked to construct a similar spreadsheet for a different initial value problem.

The last investigation explores the Taylor and MacLaurin Series (Figure 5). By using sliders to input the degree and central point of a polynomial, students explore the accuracy of the polynomial for various functions over different intervals. They also investigate how the error of the approximation is affected by the position of the centre of the series. The program shows how the Taylor and MacLaurin polynomials converge to the original function as the degree of the polynomial increases. 


\section{Evaluation and discussion}

Our laboratory program of investigations provided students with the opportunity to think more deeply about some of the mathematical concepts developed in the unit, and to explore mathematical ideas more fully. From this point of view, our aim to enhance conceptual understanding was fulfilled. A major feature of the laboratory program was its use of both visualisation and numerical methods.

In an attempt to measure the success of the laboratory program, students were surveyed at the completion of the unit. However, as only 17 students were enrolled in the unit and only nine of these completed the survey, it was not possible to reach definitive conclusions. Comparisons could not be made between the trial group and a control group as only one class was run in the unit. However, it was still informative to analyse and discuss the responses, as indications of the successes of the laboratory program became evident and concerns were highlighted.

The survey results, summarised in Table 1, indicate the level of agreement of the nine students with the various statements of the survey. Percentages have been used for ease of comparison.

An encouraging comment returned in the survey was that the program was "very helpful as practical sessions [make it] much easier to remember concepts". A majority (56\%) agreed with the statement that the program provided students with the opportunity to explore concepts discussed in lectures. This was somewhat lower than anticipated, considering the aims of the program. Perhaps students did not understand what 'exploring concepts' meant and a paraphrasing of the question may have solicited a different response. There was stronger agreement (89\%) with the statement that the program provided students with the opportunity to see how technology could be used to solve problems. This indicated that students appreciated the use of technology within the course. 
TABLE 1: Survey results in percentages of each response (of the total of nine students): $\mathrm{SA}=$ Strongly agree; $\mathrm{A}=$ Agree; $\mathrm{D}=$ Disagree; $\mathrm{SD}=$ Strongly disagree; UJ=Unable to judge.

\begin{tabular}{lrrrrr}
\hline Statement & SA & A & D & SD & UJ \\
\hline $\begin{array}{l}\text { An aim of the practical program was to pro- } \\
\text { vide resources to explore some of the concepts }\end{array}$ & 33 & 22 & 33 & 11 & 0 \\
discussed in lectures. This aim was fulfilled. & & & & & \\
\hline Another aim of the practical program was to & 22 & 67 & 11 & 0 & 0 \\
provide resources to explore how technology & & & & & \\
can be used to solve problems. This aim was & & & & & \\
fulfilled. & & & & & \\
\hline
\end{tabular}

\begin{tabular}{lllllll}
\hline $\begin{array}{l}\text { The practical classes provided a suitable learn- } \\
\text { ing activity for this unit. }\end{array}$ & 0 & 67 & 22 & 11 & 0 \\
\hline $\begin{array}{l}\text { The practical reports were a suitable form of } \\
\text { ongoing assessment in this unit. }\end{array}$ & 33 & 56 & 11 & 0 & 0 \\
\hline $\begin{array}{l}\text { The workload generated by the practical pro- } \\
\text { gram was appropriate for this unit. }\end{array}$ & 11 & 56 & 33 & 0 & 0 \\
\hline $\begin{array}{l}\text { The feedback on my practical reports was } \\
\text { helpful and timely. }\end{array}$ & 11 & 67 & 11 & 0 & 11 \\
\hline
\end{tabular}


Other indicators of student satisfaction shown in the survey included:

- $67 \%$ of the respondents said that the program was a suitable learning activity for the unit;

- $89 \%$ indicated that the laboratory reports were a suitable form of ongoing assessment;

- $67 \%$ agreed that the workload was appropriate for the unit;

- $78 \%$ indicated that they were pleased with the feedback on their reports;

- A student expressed gratification for being "given a little longer than one week to attempt the questions" so that they could manage their own time.

Participation in the program was high, with all nine respondents indicating that they attended all or nearly all of the twelve sessions. This is reflected in the fact that thirteen of the seventeen students submitted at least five of the six required practical reports. This fact was not surprising considering that $20 \%$ of the overall assessment in the unit was based on the laboratory reports.

Some negative comments were expressed in the open ended questions of the survey. A student expressed a preference for the laboratory time to be used for discussion of tutorial questions. Another's comment was: "Make them [laboratory questions] more applied to concepts. I feel they didn't really help learn components of [the] unit". Such responses indicate a need to communicate the benefits of a laboratory program, and that a thorough understanding of concepts is just as important as learning algorithms for solving problems.

An attempt was made to compare examination results of students in the 2008 class with those from previous years. However, it was not possible to draw any conclusions, as the many factors affecting performance could not be isolated. These included students' attendance, previous mathematical expe- 
riences and abilities, examination variation over the years and the effect of previous laboratory programs (some of which included mathematical investigations).

A more positive attitude towards the laboratory program was evident amongst the 2008 students compared with students from previous years. Students enrolled in programs prior to 2008 expressed concern that the laboratory program based on Maple neither helped them pass the examination, nor provided relevant software practice for their chosen career.

The 2008 program did not aim to teach CAS programming skills. However, students were encouraged to use Maple as a mathematical word processor to write up their laboratory reports. Some students showed an interest in Maple's capabilities and explored its use as a mathematical tool. Thus, it was possible for students to work at various levels of CAS competencies, as was the case in Franco's study [2].

Although our program primarily used existing multimedia resources, it is possible to implement a similar investigative program based on a CAS. The advantages of using existing resources include: the relative ease of setting up the program; the fact that the programs have been tested and are relatively trouble free; and the ability to modify the questions posed in a source. The fact that web pages change over time and are sometimes unavailable is a clear disadvantage of using web based resources. We found no such problems with availability throughout our laboratory program. Whether a laboratory program uses a CAS or web based resources, it is possible to arrange for answer submission via a web program, such as Blackboard, where instant feedback can be provided. The initial set-up time required can be recouped by the marking time saved, especially for large classes.

If scheduled class time is not available for the investigations, it is possible to set them as projects. This may be a consideration for educators who wish to avail their students of the advantages of the program without either cutting existing content, or increasing contact hours. Group work would be appropriate for projects. If the deletion of content is being contemplated, 
Cunningham suggested that "manual symbol manipulation and calculation work are becoming less productive and appropriate than they once were; probably these are good candidates for replacement" [1, p.72].

\section{Conclusion}

Our trial revealed that many students view mathematics as learning a set of algorithms for solving familiar problems, so as to pass the examination. Our laboratory program has gone some way towards helping students to internalise mathematical concepts, so that they can be used in new situations that may arise in the non-immediate future. These potential benefits may not be available to students following laboratory programs that aim to skill students in a particular CAS. Our trial has also exposed the need to communicate to students the importance of fully understanding mathematical concepts and that mathematics involves more than solving familiar problems via a learnt algorithm.

Further research into the development of students' understanding of mathematical concepts through the use of investigative laboratory programs is needed. We plan to expand upon our present trial by using a larger population, in order to compare results against a control group. The effects of the program on such things as performance on both skill and concept examination questions, on students' ability to use mathematical concepts in unfamiliar situations and on their ability to retain mathematical concepts over a period of time, are all worthy of further investigation. The development of a laboratory program of mathematical investigations based on the use of a CAS, as distinct from multimedia or hypermedia resources, is also worthy of investigation. 


\section{References}

[1] S. Cunningham. The visualization environment for mathematics education. In Visualization in Teaching and Learning Mathematics, ed. W. Zimmermann and S. Cunningham, 67-76. USA, Mathematical Association of America, 1991. C282, C285, C296

[2] A. Franco, P. Franco, A. Garcia, F. Garcia, F. J.Gonzalez, S. Hoya, G. Rodriguez, and A. de la Villa. Learning calculus of several variables with new technologies. The International Journal of Computer Algebra in Mathematics Education, 7 (4), 295-309, 2000. C284, C295

[3] B. E. Garner and L. E. Garner. Retention of concepts and skills in traditional and reformed applied calculus. Mathematics Education Research Journal, 13 (3), 165-184, 2001 C282

[4] S. Habre. Visualization enhanced by technology in the learning of multivariate calculus. The International Journal of Computer Algebra in Mathematics Education, 8 (2), 115-130, 2001. C282, C283

[5] B. H. Hallet. Visualization and calculus reform. In Visualization in Teaching and learning Mathematics, ed. W. Zimmermann and S. Cunningham, 121-126, 1991. USA, Mathematical Association of America C281, C283

[6] F. Marton and R. Saljo. Approaches to learning. In eds. F. Marton, D. Hounsell and N. Entwistle, The Experience of Learning, 36-55, 1984. Scottish Academic Press, Edinburgh. C282

[7] R. Moreno and R. Mayer. Verbal redundancy in multimedia learning; When reading helps listening. Journal of Educational Psychology, 94 (1), 153-163, 2002. C282

[8] L. D. Murphy. Computer algebra systems in calculus reform, MSTE, University of Illinois at Urbana-Champaign, 1999. http://mste . illinois.edu/users/Murphy/Papers/CalcReformPaper.html C282 
[9] M. Pemberton. Integrating web-based maple with a first year calculus and linear algebra course. Proceedings of the 2nd International Conference on the Teaching of Mathematics, Hersonissos, Greece, July 2002. http://www. math.uoc.gr/ ictm2/Proceedings/pap316.pdf $\mathrm{C} 284$

[10] R. Pierce and K. Stacey. Observations on students' responses to learning in a CAS environment. Mathematics Education Research Journal, 13 (1), 28-46, 2001. C282

[11] M. D. Roblyer. Integrating Educational Technology Into Teaching (4th Ed.), 2006. Pearson, New Jersey, USA. C282

[12] J. Stewart. Calculus (5th Ed.), 2003. Brooks/Cole, Belmont, USA. $\mathrm{C} 285$

[13] E. J. Tonkes, B. I. Loch and A. W. Stace. An innovative learning model for computation in first year mathematics. International Journal of Mathematical Education in Science and Technology, 36 (7), 751-759, 2005. C284

[14] L. M. Villarreal. A step in the positive direction: Integrating a computer laboratory component into developmental algebra courses. Mathematics and Computer Education, 37 (1), 72-78, 2003. C283

[15] S. Vinner. The pseudo-conceptual and the pseudo-analytical thought processes in mathematics learning. Educational Studies in Mathematics, 34 (2), 97-129, 1997. C285

[16] P. Vlachos and A.K. Kehagias. A computer algebra system and a new approach for teaching business calculus. The International Journal of Computer Algebra in Mathematics Education. 7 (2), 87-104, 2000. C282, C284 


\section{Author address}

1. Raymond Summit, Western Australian School of Mines, Curtin University of Technology, Western Australia, Australia. mailto:r.summit@curtin.edu.au 